Series A

\author{
I. MATHEMATICA
}

316

\title{
A CONDITION \\ FOR THE SELFADJOINTNESS OF A LINEAR OPERATOR
}

BY

TOIVO NIEMINEN

HEL S I K I 1962

S U O M A L A I N E T I E D E A K A T EMIA 
Communicated 9 April 1962 by R. and F. Nevanlinna 


\section{A condition for the selfadjointness of a linear operator}

Let $T$ be a selfadjoint linear operator from a complex Hilbert space $\mathfrak{G}$ into $\mathfrak{G}$. It is well known that its spectrum lies on the real axis and that its resolvent

$$
R_{\lambda}=(T-\lambda I)^{-1} \quad(I=\text { the identity operator })
$$

satisfies the inequality

$$
\left|R_{\dot{\lambda}}\right| \leqq \frac{1}{|\operatorname{Im} \lambda|}
$$

for every $\lambda$ with $\operatorname{Im} \lambda \neq 0$. The purpose of this paper is to prove the following converse theorem:

Let $T$ be a linear operator from a complex Hilbert space $\mathfrak{\mathfrak { B }}$ into $\mathfrak{\mathfrak { G }}$ such that

(a) the domain of definition $\mathfrak{D}_{T}$ of $T$ is dense in $\mathfrak{H}$,

(b) the imaginary axis, with the possible exception of the origin, belongs to the resolvent set of $T$,

(c) the resolvent of $T$ satisfies the inequality

$$
\left|R_{i \xi}\right| \leqq \frac{1}{|\xi|}
$$

$$
\text { for every real } \xi \neq 0 \text {. }
$$

Then $T$ is selfadjoint.

Proof. The inequality in (c) can be written

$$
|T x-i \xi x|^{2} \geqq|\xi|^{2}|x|^{2} .
$$

Since here

$$
|T x-i \xi x|^{2}=|T x|^{2}+|\xi|^{2}|x|^{2}+i \xi[(T x, x)-(x, T x)],
$$

we thus have

or

$$
|T x|^{2}+i \xi[(T x, x)-(x, T x)] \geqq 0
$$

$$
|T x|^{2} \geqq 2 \xi \operatorname{Im}(T x, x)
$$


for every $x$ in $\mathfrak{D}_{T}$ and every real $\xi$. From this it immediately follows that $\operatorname{Im}(T x, x)=0$ or

$$
(T x, x)=(x, T x)
$$

for every $x$ in $\mathfrak{D}_{T}$. By means of the polarization formula

$$
\begin{aligned}
4(T x, y)= & (T(x+y), x+y)-(T(x-y), x-y)+ \\
& i(T(x+i y), x+i y)-i(T(x-i y), x-i y)
\end{aligned}
$$

and the corresponding formula for $(x, T y)$ we thus get

$$
(T x, y)=(x, T y)
$$

for every $x$ and $y$ in $\mathfrak{D}_{T}$. This shows that the adjoint operator $T^{*}$, which exists by virtue of (b), is an extension of $T$.

In order to prove conversely that $T$ is an extension of $T^{*}$ we first show that $R_{i}^{*}=R_{-i}$. From (1) it follows that

$$
(T x-i x, y)=(x, T y+i y)
$$

whenever $x$ and $y$ are in $\mathfrak{D}_{T}$. Denoting here

$$
T x-i x=u, \quad T y+i y=v, \quad x=R_{i} u, \quad y=R_{-i} v
$$

we thus get

$$
\left(u, R_{-i} v\right)=\left(R_{i} u, v\right)
$$

for every $u$ and $v$ in $\mathfrak{B}$. This shows that $R_{i}^{*}=R_{-i}$.

Now let $y$ be an arbitrary vector of $\mathscr{D}_{T^{*}}$. If we set $T^{*} y=z$ we thus have

$$
(T x, y)=(x, z)
$$

and, consequently,

$$
(T x-i x, y)=(x, z+i y)
$$

for every $x$ in $\mathfrak{D}_{T}$. Denoting here

$$
T x-i x=u, \quad x=R_{i} u
$$

we therefore see by virtue of the equation (2) that

$$
(u, y)=\left(R_{i} u, z+i y\right)=\left(u, R_{-i}(z+i y)\right) .
$$

Since $u$ is arbitrary, we thus have

$$
y=R_{-i}(z+i y) \in D_{T+i I}=D_{T} .
$$

As a consequence, $\mathfrak{D}_{T^{*}} \subset \mathfrak{D}_{T}$, and our proof is complete. 
It is well known that the spectrum of a unitary operator $U$ lies on the unit circle and that its resolvent satisfies the inequality

$$
\left|R_{\lambda}\right| \leqq \frac{1}{|| \lambda|-1|}
$$

It would be interesting to know whether from these two assumptions it conversely follows that $U$ is unitary. 